\section{Surgical outcomes of three different surgical techniques for treatment of convergence insufficiency intermittent exotropia}

\begin{abstract}
Purpose To determine the outcomes of three different techniques of strabismus surgery in patients with convergence insufficiency intermittent exotropia (CI-X(T)). Patients and methods Sixty-seven patients with CI-X(T) with near-distance disparity (NDD) $\geq 10$ prism diopter (PD) were included in this 1-year follow-up prospective study and were randomly divided into three groups: slanted bilateral LR recession (S-BLR) group in which 22 patients underwent bilateral slanting recession of the lateral rectus (LR) muscle, the I-RR group with 23 patients who underwent improved unilateral medial rectus (MR) resection and LR recession with the amounts of resection and recession biased to near and distance deviation, respectively, and the A-BLR group with 22 patients who underwent bilateral augmented LR recession based on the near deviation. A successful outcome at distant and near was defined as exodeviation between 10 PD of exophoria/ tropia and 5 PD of esophoria/tropia. Cumulative probabilities of success, preoperative and postoperative distant, near deviations, and NDD among groups were analyzed and compared.

Results The success rate of distant exodeviation, near exodeviation, and NDD in the three groups after 1 year was statistically insignificant $(P=0.054,0.233$, and 0.142 , respectively). At the 1 year follow-up, vertical pattern strabismus ( $\mathrm{V}$ and $\mathrm{A}$ patterns) was a feature of the S-BLR group, whereas the rate of postoperative overcorrection and undercorrection was significant in the A-BLR and I-RR groups, respectively.

Conclusion The success rate of correction of distant exodeviation, near exodeviation, and

NDD was statistically indifferent among the three groups. However, each procedure has its specific postoperative concerns, which should be considered before implementing in patients with $\mathrm{CI}-\mathrm{X}(\mathrm{T})$.

Eye (2018) 32, 693-700; doi:10.1038/eye.2017.259; published online 22 December 2017

\section{Introduction}

Convergence insufficiency intermittent exotropia $(\mathrm{CI}-\mathrm{X}(\mathrm{T}))$ is a rare form of intermittent exotropia characterized by an exodeviation greater at near fixation than at distance by 10 prism diopters (PD) or more. ${ }^{1}$ Success rate of various surgical modalities has been reported to range from 18 to $92 \%$ and, therefore, there is a current trend for optimization of surgical strategies to achieve better outcomes. ${ }^{2}$ The different surgical options for CI-X(T) include bilateral lateral rectus (LR) recession, ${ }^{3}$ bilateral medial rectus (MR) resection, ${ }^{4,5}$ unilateral MR resection, ${ }^{6}$ conventional unilateral LR recession with MR resection, ${ }^{7}$ slanting procedures ${ }^{8,9}$ and adjustable sutures. ${ }^{10}$

In the surgical armamentarium of $\mathrm{CI}-\mathrm{X}(\mathrm{T})$, some authors proposed using the slanting recession of the LR muscle, in which, the lower fibers of the LR muscle are recessed more than its upper fibers with resultant greater correction of near deviation than on the distant one. ${ }^{8}$ Other surgeons proposed augmenting the LR recession according to near measurements-or even more — for treatment of CI-X(T). ${ }^{11}$ Kraft et al ${ }^{12}$ in 1995 introduced the modality of improved unilateral recession-resection surgery for treatment of CI-X (T), in which the surgical formula was biased to strengthen the MR more than weakening of the LR by resecting the MR according to near
\end{abstract}

MF Farid ${ }^{1,2}$ and EA Abdelbaset ${ }^{2}$

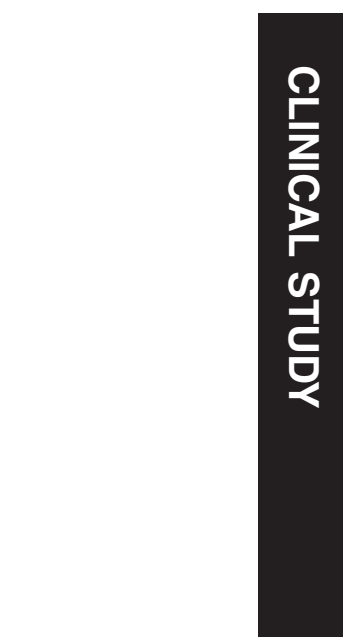

${ }^{1}$ Department of Ophthalmology, Benha Faculty of Medicine, Benha University, Benha, Egypt

${ }^{2}$ Department of Ophthalmology, Benha University Hospital, Benha, Egypt

Correspondence: MF Farid, Department of Ophthalmology, Benha Faculty of Medicine, Benha University, 1 Farid Nada Street New Benha, Benha, Benha, Qualyobia 13511, Egypt

Tel: +002010033 546 36.

Fax: +013 322766 .

E-mail: Mohamed_fathy_ 10@yahoo.com

Received: 11 September 2017

Accepted in revised form: 16 October 2017

Published online: 22

December 2017 
deviation and recessing the LR according to the distant one. ${ }^{11,12}$ In order to find the best surgical option for this rare subtype of intermittent $\mathrm{XT}$, we have conducted this comparative prospective trial between these three different surgical strategies.

\section{Patients and methods}

This prospective study included 67 consecutive patients with CI-X(T) and was conducted between June 2012 and April 2016. Before surgery, informed written consent was obtained from adult patients and from parents/guardians of the pediatric patients according to the approved protocol by the Medical Ethics Committee of Benha University Hospital, Benha Faculty of Medicine for the protection of human subjects (Declaration of Helsinki). Patients with CI-X(T) (near XT that exceeds distant XT by $\geq 10 \mathrm{PD}$ ) after $24 \mathrm{~h}$ monocular patching, which was confirmed at least at two repeated examinations, 2 weeks apart, were included in the study. The minimum age for inclusion was 4 years. Patients with amblyopia, paralytic or restrictive strabismus, previous strabismus surgeries, A or $\mathrm{V}$ pattern, oblique muscle overaction, congenital anomalies, or neurological disorders were excluded from the study.

A detailed history was obtained from all patients including age of onset and the daily duration of deviation. Constant deviation was defined as exodeviation for $100 \%$ of waking hours. Complete ophthalmic and orthoptic examinations were then performed including best corrected visual acuity, cycloplegic refraction, motility assessment, anterior segment, and fundus examinations. Patients with hyperopia $>3.00$ diopter (D) were given glasses $\sim 1.00-1.50 \mathrm{D}$ less than their full cycloplegic hyperopic refraction while patients with myopia $\geq 1.00 \mathrm{D}$, astigmatism $\geq 1.50 \mathrm{D}$ were given glasses, which fully corrected their myopia or astigmatism. Angle of deviation before surgery and at each postoperative visit was measured by prism and alternate cover test (PACT) with accommodative targets for fixation at $1 / 3$ and $6 \mathrm{~m}$. Preoperatively, an additional measurement was obtained after $24 \mathrm{~h}$ monocular patching of the habitually deviating eye, and post-occlusion measurements were taken before allowing the patient to regain binocular fusion. According to the difference between distant and near deviations after monocular occlusion, $X(T)$ was classified based on the Burian's classification system ${ }^{1,7}$ and only the CI-X(T) type was included in this study. According to the classification system for CI-X(T) introduced by Yang and Hwang, ${ }^{11}$ patients were further classified according to their response to monocular occlusion testing; patients with true $\mathrm{CI}-\mathrm{X}(\mathrm{T})$; near $\mathrm{X}(\mathrm{T})>$ distant $\mathrm{X}(\mathrm{T})$ by more than $10 \mathrm{PD}$ both before and after monocular occlusion, together with patients with masked CI-X(T); near $X(T)>$ distant $X(T)$ by more than 10 PD only after monocular occlusion were included in the study. Patients with pseudo CI-X(T), near $X(T)>$ distant $X(T)$ by more than 10 PD only before but not after monocular occlusion test were excluded from the study. The AC/A ratio was measured using the gradient method. ${ }^{13}$ Fusion was first assessed with worth four-dot test and then near stereoacuity in current refractive correction was measured with Titmus fly stereotest and stereoacuity of $<100 \mathrm{~s}$ of arc was defined as good. Postoperative, improved stereopsis was defined as a decrease of $\geq 2$ octaves at the last follow-up visit or before reoperation, and decreased stereopsis was defined as an increase of $\geq 2$ octaves. ${ }^{14}$ Patients were counseled before operation that in cases of postoperative overcorrection at distance and/or at near, temporary alternate patching or prism therapy would be required.

According to the different surgical procedures, all patients were divided into three groups: group (1) slanted bilateral LR recession (S-BLR) group in which the upper horn of the muscle was recessed based on the distance exodeviation while the lower horn was recessed based on the near exodeviation, ${ }^{8}$ group (2); improved unilateral $M R$ resection and LR recession (I-RR) in which the MR resection is based on the near deviation, while the LR recession is based on the distant deviation ${ }^{12}$ and group (3); augmented bilateral LR recession (A-BLR) group in which LR recession was augmented to the near exodeviation. Patients with ocular dominance were directed to recession-resection group and the surgery was performed on the non-dominant eye or the more often exotropic eye. Patients without ocular dominance were randomized to either slanted or augmented groups. The magnitude of deviation for which to perform surgery was the largest preoperative deviation measured after $24 \mathrm{~h}$ monocular occlusion at distance and near fixation by PACT.

All surgeries were performed under general anesthesia using the same surgical table and were performed by single author (MFF) together with an assistant using conventional limbal conjunctival incision. For LR recession, it was measured from the back of the insertion of the muscle after muscle disinsertion and the muscle was sutured directly to the globe. For MR resection, the measurement was made from the insertion of the muscle prior to muscle disinsertion. For LR slanting recession, the muscle was reinserted in an oblique manner relative to its original insertion: ${ }^{8}$ the upper horn of the muscle was recessed according to the distant exodeviation (range: $4-7.5 \mathrm{~mm}$ ), and the lower horn was recessed according to near exodeviation (range; $6-11 \mathrm{~mm}$ ). Postoperative examinations were performed by an orthoptist who was masked to the surgery performed and were scheduled at 1 week and 1, 3, 6, and 12 months. They included 
Table 1 Patients' characteristics

\begin{tabular}{|c|c|c|c|c|}
\hline Characteristics, mean \pm s.d. & $S-B L R$ & $I-R R$ & $A-B L R$ & P-value \\
\hline Age at surgery (years) & $15.6 \pm 12.02$ & $17.3 \pm 12.3$ & $16.2 \pm 7.04$ & $0.865^{\mathrm{a}}$ \\
\hline Gender, male & $16(72.7 \%)$ & $11(47.8 \%)$ & $17(77.2 \%)$ & $0.08^{\mathrm{b}}$ \\
\hline Refractive error OD, (S.E.; D) & $-0.35 \pm 1.2$ & $-1.3 \pm 2.1$ & $-0.6 \pm 1.9$ & $0.186^{\mathrm{a}}$ \\
\hline Refractive error OS, (S.E.; D) & $-0.17 \pm 1.5$ & $-1.2 \pm 2.08$ & $0.5 \pm 1.9$ & $0.168^{\mathrm{a}}$ \\
\hline BCVA, OD (logMAR) & $1.02 \pm 0.2$ & $0.9 \pm 0.1$ & $1.01 \pm 0.3$ & $0.122^{\mathrm{a}}$ \\
\hline BCVA, OS (logMAR) & $1.06 \pm 0.2$ & $1.03 \pm 0.3$ & $1.02 \pm 0.3$ & $0.878^{\mathrm{a}}$ \\
\hline Distance exodeviation $(\Delta)$ & $28.4 \pm 10.8$ & $33.7 \pm 15.9$ & $24.4 \pm 10.2$ & $0.052^{\mathrm{a}}$ \\
\hline Near exodeviation $(\Delta)$ & $46.3 \pm 13.8$ & $46.4 \pm 16.1$ & $37.6 \pm 9.9$ & $0.053^{\mathrm{a}}$ \\
\hline Near-distance difference $(\Delta)$ & $17.9 \pm 8.6$ & $12.7 \pm 7.8$ & $13.2 \pm 6.9$ & $0.056^{\mathrm{a}}$ \\
\hline Preoperative good stereopsis & $10 / 22(45.4 \%)$ & $14 / 23(60.8 \%)$ & $13 / 22(59.09 \%)$ & $0.527^{\mathrm{b}}$ \\
\hline Postoperative good stereopsis & $14 / 22(63.6 \%)$ & $18 / 23(78.2 \%)$ & $17 / 22(77.2 \%)$ & $0.470^{\mathrm{b}}$ \\
\hline Improved stereopsis & $8 / 22(36.3 \%)$ & $12 / 23(52.1 \%)$ & $13 / 22(59.09 \%)$ & $0.302^{\mathrm{b}}$ \\
\hline Stationary stereopsis & $7 / 22(31.8)$ & $9 / 23(39.1 \%)$ & $6 / 22(27.2 \%)$ & $0.693^{\mathrm{b}}$ \\
\hline Worsened stereopsis & $7 / 22(31.8)$ & $2 / 23(8.6 \%)$ & $3 / 22(13.6 \%)$ & $0.105^{\mathrm{b}}$ \\
\hline Constant deviation & $9 / 22(40.9 \%)$ & $21 / 23(91.3 \%)$ & $5 / 22(22.7 \%)$ & $0.000^{\mathrm{b}, \mathrm{c}}$ \\
\hline Pre-op AC/A ratio & $1.82 \pm 0.86$ & $1.85 \pm 0.52$ & $2.13 \pm 0.36$ & $0.189^{\mathrm{a}}$ \\
\hline Post-op AC/A ratio & $2.04 \pm 1.87$ & $2.6 \pm 0.8$ & $2.06 \pm 0.5$ & $0.214^{\mathrm{a}}$ \\
\hline Initial undercorrection (distant) & $8 / 22(36.3 \%)$ & $14 / 23(60.8 \%)$ & $0 / 22$ & $0.000^{\mathrm{b}, \mathrm{c}}$ \\
\hline Initial undercorrection (near) & $13 / 22(59.09 \%)$ & $13 / 23(56.5 \%)$ & $4 / 22(18.1 \%)$ & $0.009^{\mathrm{b}, \mathrm{c}}$ \\
\hline Final undercorrection (distant) & $5 / 22(22.7 \%)$ & $13 / 23(56.5 \%)$ & $5 / 22(22.7 \%)$ & $0.021^{\mathrm{b}, \mathrm{c}}$ \\
\hline Final undercorrection (near) & $11 / 22(50 \%)$ & $16 / 23(69.5 \%)$ & $16 / 22(72.7 \%)$ & $0.233^{\mathrm{b}}$ \\
\hline Initial overcorrection (distant) & $5 / 22(22.7 \%)$ & $0 / 23(0 \%)$ & $8 / 22(36.3 \%)$ & $0.007^{\mathrm{b}, \mathrm{c}}$ \\
\hline Initial overcorrection (near) & $0 / 22$ & $0 / 23$ & $0 / 22$ & $\mathrm{~N} / \mathrm{A}$ \\
\hline Final overcorrection (distant) & $0 / 22$ & $0 / 23$ & $6 / 22(27.2 \%)$ & $0.001^{\mathrm{b}, \mathrm{c}}$ \\
\hline Final overcorrection (near) & $0 / 22$ & $0 / 23$ & $0 / 22$ & $\mathrm{~N} / \mathrm{A}$ \\
\hline
\end{tabular}

Abbreviations: $\Delta$, prism diopter; A-BLR, augmented bilateral lateral rectus recession; ANOVA, analysis of variance; $\mathrm{D}$, diopter; I-RR, improved unilateral recession-resection; preop, preoperative; post-op, postoperative; S-BLR, slanting bilateral lateral rectus recession; SE, spherical equivalent. ${ }^{a} P$-value by univariate ANOVA test. ${ }^{b} P$-value by $\chi^{2}$. ${ }^{c}$ Significant $P$-value.

alignment in the primary position at distance and near and Titmus stereotest. AC/A ratio was assessed postoperatively only at the last follow-up visit.

A successful outcome was defined as a postoperative residual deviation at near and distance between 10 PD of exophoria/tropia and 5 PD of esophoria/tropia with a near-distance difference of $<10$ PD. Postoperative consecutive esotropia $>5$ PD at first week was considered as an overcorrection and was initially managed by full cycloplegic hypermetropic prescription together with alternating full-time monocular patching. Consecutive esotropia persisted more than 1 month was treated by press-on base-out Fresnel prism (3M Health Care, St. Paul, MN) to allow constant fusion until full resolution of esotropia and, if esotropia persisted for 3 months, the prism could be incorporated into patient's glasses. Patients with persistent or increasing esotropia of more than 15 PD after 6 months despite all previous measures were reoperated. Postoperative exotropia of more than 10 PD at distant and/or near was considered as recurrence. Initially, those patients were treated by non-surgical measures such as part-time occlusion or minus-lens therapy and reoperation was offered for patients with constant exotropia $\geq 14$ PD at distant and/or near after 6 months.
Patients reoperated for postoperative consecutive esotropia, A-V pattern, or recurrent exotropia before the final postoperative visit at 1 year were excluded from final data analysis.

All analyses were performed with statistical software (StatLab, SPSS for Windows V.17.0). Paired t-test and Univariate analysis of variance were used to compare preoperative and postoperative numerical data among the groups while a $\chi^{2}$-test was used to compare the nominal values among groups. A $P$-value of $<0.05$ was considered statistically significant.

\section{Results}

During the study period, a total of 67 patients met the inclusion criteria and were divided into three groups: the S-BLR group that included 22 patients (32.8\%), the I-RR group with 23 patients $(34.3 \%)$, and the A-BLR group with 22 patients (32.8\%). In our patient cohort, the symptoms of CI-X(T) were outward deviation in all 67 patients, photophobia in 13 patients, and diplopia in five patients. Preoperatively, none of the patients had worn prism glasses. Push-up training was attempted to build convergence fusional amplitude in 37 patients $(55.2 \%)$, but with no effect 
Table 2 Surgical outcomes of the three groups at 12 months

\begin{tabular}{|c|c|c|c|c|c|c|}
\hline Group & Surgical results & 1 Day & 1 Month & 3 Months & 6 Months & 12 Months \\
\hline \multicolumn{7}{|l|}{ Distant } \\
\hline \multirow[t]{3}{*}{ S-BLR $(n=22)$} & Success & $9 / 22(40.9 \%)$ & $9 / 22(40.9 \%)$ & $9 / 22(40.9 \%)$ & $14 / 22(63.6 \%)$ & $17 / 22(77.2 \%)$ \\
\hline & Undercorrection & $8 / 22(36.3 \%)$ & $5 / 22(22.7 \%)$ & $4 / 22(18.1 \%)$ & 4/22 (18.1\%) & $5 / 22(22.7 \%)$ \\
\hline & Overcorrection & $5 / 22(22.7 \%)$ & $8 / 22(36.3 \%)$ & $9 / 22(40.9 \%)$ & $4 / 22(18.1 \%)$ & $0 / 22(0 \%)$ \\
\hline \multirow[t]{3}{*}{ I-RR $(n=23)$} & Success & $9 / 23(39.1 \%)$ & $8 / 23(34.7 \%)$ & $9 / 23(39.1 \%)$ & $8 / 23(34.7 \%)$ & $10 / 23(43.4 \%)$ \\
\hline & Undercorrection & $14 / 23(60.8 \%)$ & $14 / 23(60.8 \%)$ & $14 / 23(60.8 \%)$ & $15 / 23(65.2 \%)$ & $13 / 23(56.5 \%)$ \\
\hline & Overcorrection & $0 / 23$ & $1 / 23(4.3 \%)$ & $0 / 23$ & $0 / 23$ & $0 / 23$ \\
\hline \multirow[t]{3}{*}{ A-BLR $(n=22)$} & Success & $14 / 22(63.6 \%)$ & $18 / 22(81.8 \%)$ & $16 / 22(72.7 \%)$ & $11 / 22(50 \%)$ & $11 / 22(50 \%)$ \\
\hline & Undercorrection & $0 / 22$ & $0 / 22(0.13 \%)$ & $0 / 22(0.18 \%)$ & $5 / 22(22.7 \%)$ & $5 / 22(22.7 \%)$ \\
\hline & Overcorrection & $8 / 22(36.3 \%)$ & $4 / 22(18.1 \%)$ & $6 / 22(27.2 \%)$ & $6 / 22(27.2 \%)$ & $6 / 22(27.2 \%)$ \\
\hline \multirow[t]{3}{*}{$P$-value of success rate } & S-BLR and I-RR & 0.903 & 0.671 & 0.903 & 0.055 & $0.022^{\mathrm{a}}$ \\
\hline & S-BLR and A-BLR & 0.136 & $0.005^{\mathrm{a}}$ & $0.035^{\mathrm{a}}$ & 0.368 & 0.063 \\
\hline & I-RR and A-BLR & 0.104 & $0.001^{\mathrm{a}}$ & $0.025^{\mathrm{a}}$ & 0.304 & 0.660 \\
\hline \multicolumn{7}{|l|}{ Near } \\
\hline \multirow[t]{3}{*}{ S-BLR $(n=22)$} & Success & $9 / 22(40.9 \%)$ & $9 / 22(40.9 \%)$ & $12 / 22(54.5 \%)$ & $9 / 22(40.9 \%)$ & $11 / 22(50 \%)$ \\
\hline & Undercorrection & $13 / 22(59.09 \%)$ & $13 / 22(59.09 \%)$ & $10 / 22(45.4 \%)$ & $13 / 22(59.09 \%)$ & $11 / 22(50 \%)$ \\
\hline & Overcorrection & $0 / 22$ & $0 / 22$ & $0 / 22$ & $0 / 22$ & $0 / 22$ \\
\hline \multirow[t]{3}{*}{ I-RR $(n=23)$} & Success & $10 / 23(43.4 \%)$ & $8 / 23(34.7 \%)$ & $16 / 23(69.5 \%)$ & $6 / 23(26.08 \%)$ & $7 / 23(30.4 \%)$ \\
\hline & Undercorrection & $13 / 23(56.5 \%)$ & $15 / 23(65.2 \%)$ & $7 / 23(30.4 \%)$ & $17 / 23(73.9 \%)$ & $16 / 23(69.5 \%)$ \\
\hline & Overcorrection & $0 / 23$ & $0 / 23$ & $0 / 23$ & $0 / 23$ & $0 / 23$ \\
\hline \multirow[t]{3}{*}{ A-BLR $(n=22)$} & Success & $18 / 22(81.8 \%)$ & $13 / 22(59.09 \%)$ & $11 / 22(50 \%)$ & $10 / 22(54.5 \%)$ & $6 / 22(27.2 \%)$ \\
\hline & Undercorrection & $4 / 22(18.1 \%)$ & $9 / 22(40.9 \%)$ & $11 / 22(50 \%)$ & $12 / 22(45.4 \%)$ & $16 / 22(72.7 \%)$ \\
\hline & overcorrection & $0 / 22$ & $0 / 22$ & $0 / 22$ & $0 / 22$ & $0 / 22$ \\
\hline \multirow[t]{3}{*}{ P-value of success rate } & S-BLR and I-RR & 0.866 & 0.671 & 0.903 & 0.297 & 0.184 \\
\hline & S-BLR and A-BLR & $0.005^{\mathrm{a}}$ & $0.005^{\mathrm{a}}$ & $0.035^{\mathrm{a}}$ & 0.372 & 0.124 \\
\hline & I-RR and A-BLR & $0.008^{\mathrm{a}}$ & $0.001^{\mathrm{a}}$ & $0.025^{\mathrm{a}}$ & 0.054 & 0.814 \\
\hline \multicolumn{7}{|l|}{$N D D$} \\
\hline S-BLR $(n=22)$ & Success & $14 / 22(63.6 \%)$ & $9 / 22(40.9 \%)$ & $9 / 22(40.9 \%)$ & $9 / 22(40.9 \%)$ & $13 / 22(59.09 \%)$ \\
\hline I-RR $(n=23)$ & Success & $23 / 23(100 \%)$ & $16 / 23(69.5 \%)$ & $8 / 23(34.7 \%)$ & $8 / 23(34.7 \%)$ & $7 / 23(30.4 \%)$ \\
\hline A-BLR $(n=22)$ & Success & $18 / 22(81.8 \%)$ & $13 / 22(59.09 \%)$ & $17 / 22(77.2 \%)$ & $16 / 22(72.7 \%)$ & $11 / 22(50 \%)$ \\
\hline \multirow[t]{3}{*}{$P$-value of success rate } & S-BLR and I-RR & $0.001^{\mathrm{a}}$ & 0.056 & 0.671 & 0.671 & 0.055 \\
\hline & S-BLR and A-BLR & 0.180 & 0.232 & $0.015^{\mathrm{a}}$ & $0.015^{\mathrm{a}}$ & 0.549 \\
\hline & I-RR and A-BLR & $0.034^{\mathrm{a}}$ & 0.470 & $0.004^{\mathrm{a}}$ & $0.004^{\mathrm{a}}$ & 0.184 \\
\hline
\end{tabular}

Abbreviations: A-BLR, augmented bilateral lateral rectus recession; I-RR, improved unilateral recession-resection; S-BLR, slanting bilateral lateral rectus recession. ${ }^{a}$ Significant $P$-value.

on the angle of deviation or symptoms. The preoperative patient characteristics, which were not significantly different among the three groups, are shown in Table 1. Cumulative probabilities of success, undercorrections, and overcorrections in the three groups are shown in Table 2.

\section{Angle of deviation}

The average of distant exodeviation, near exodeviation and near-distant disparity preoperatively and at each postoperative follow-up visit are displayed in Table 3 and Figure 1. At 1 year after surgery, the cumulative probabilities of success at distant fixation, near fixation and NDD were not statistically significant among groups $(P$-value $=0.054,0.233$ and 0.142 respectively).

\section{Distant exodeviation}

In all groups, the mean distance exodeviation showed a significant postoperative reduction $(P<0.001$, paired $t$ test). The cumulative probability of success of distant exodeviation was $77.2 \%, 43.4 \%$, and $50 \%$ in the S-BLR, I-RR, and A-BLR groups, respectively, with a difference that approaches statistical significance $(P=0.054$;

Figure 2a). The highest rate of undercorrection of distant exodeviation at 1 year postoperative was recorded in I-RR group with $56.5 \%$ compared with $22.7 \%$ for each of the other groups, and the difference was clinically significant $(P=0.021)$. Cases with overcorrection (consecutive esotropia) at distant fixation that persisted till the last follow-up visit were recorded only in the A-BLR group (six cases, $27.2 \%$ ), a feature that was found significant compared with S-BLR $(P=0.009)$ and I-RR groups, respectively $\left(P=0.007, \mathrm{~N}-1 \chi^{2}\right.$-test; Table 2$)$. 
Table 3 Pre- and postoperative angle of deviation

\begin{tabular}{cccccccc}
\hline Deviation & Groups & Preop angle $(\Delta)$ & 1 Day $(\Delta)$ & 1 Month $(\Delta)$ & 3 Months $(\Delta)$ & 6 Months $(\Delta)$ & 12 Months $(\Delta)$ \\
\hline D & S-BLR & $28.4 \pm 10.8$ & $5.8 \pm 7$ & $2.6 \pm 11.08$ & $1.2 \pm 11.6$ & $3.2 \pm 10.8$ & $6.4 \pm 8.2$ \\
& I-RR & $33.7 \pm 15.9$ & $7.01 \pm 8.7$ & $4.6 \pm 10.01$ & $6.09 \pm 7.9$ & $6.9 \pm 6.5$ & $8.6 \pm 4.6$ \\
& A-BLR & $24.4 \pm 10.2$ & $-1.4 . \pm 9.9$ & $2.6 \pm 10.08$ & $2.5 \pm 9.8$ & $4.5 \pm 9.8$ & $4.5 \pm 10.1$ \\
& P-value & 0.052 & $0.003^{\mathrm{a}}$ & 0.757 & 0.232 & 0.395 & 0.229 \\
N & S-BLR & $46.3 \pm 13.8$ & $11.9 \pm 5.3$ & $13 \pm 11.2$ & $9.8 \pm 7.2$ & $13.8 \pm 7.7$ & $16.2 \pm 8.3$ \\
& I-RR & $46.4 \pm 16.1$ & $10.3 \pm 11.2$ & $10.4 \pm 15.5$ & $15.3 \pm 9.1$ & $18.1 \pm 6.3$ & $18.3 \pm 7.2$ \\
& A-BLR & $37.6 \pm 9.9$ & $3.4 \pm 7.4$ & $11 \pm 9.05$ & $10.5 \pm 8.2$ & $11.7 \pm 10.2$ & $15.2 \pm 12.2$ \\
& P-value & 0.053 & $0.003^{\mathrm{a}}$ & 0.761 & 0.057 & $0.034^{\mathrm{a}}$ & 0.534 \\
NDD & S-BLR & $17.9 \pm 8.6$ & $6.1 . \pm 4.2$ & $10.4 . \pm 11.4$ & $8.6 \pm 9.4$ & $10.6 \pm 9.2$ & $9.8 \pm 8.2$ \\
& I-RR & $12.7 \pm 7.8$ & $3.2 . \pm 4.2$ & $5.8 \pm 12.7$ & $9.2 \pm 8.05$ & $11.2 \pm 6.4$ & $9.7 \pm 5.9$ \\
& A-BLR & $13.2 \pm 6.9$ & $6.4 . \pm 4.8$ & $8.4 \pm 9.5$ & $8 . \pm 9.1$ & $7.2 . \pm 10.2$ & $5.3 \pm 11.1$ \\
& P-value & 0.056 & $0.032^{\mathrm{a}}$ & 0.396 & 0.902 & 0.263 & 0.149 \\
\hline
\end{tabular}

Abbreviations: $\Delta$, prism diopter; A-BLR, augmented bilateral lateral rectus recession; $\mathrm{D}$, deviation at distance; I-RR, improved unilateral recessionresection; $\mathrm{N}$, deviation at near; NDD, near-distant difference; preop, preoperative exotropia; S-BLR, slanting bilateral lateral rectus recession. $P$-value by one-way analysis of variance. ${ }^{a}$ Significant $P$-value; (-) sign, angle of esotropic deviation.

a

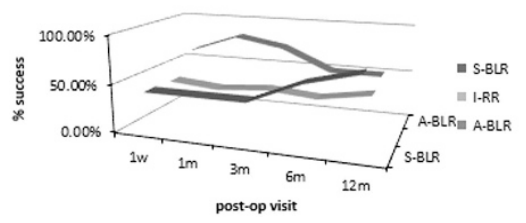

b

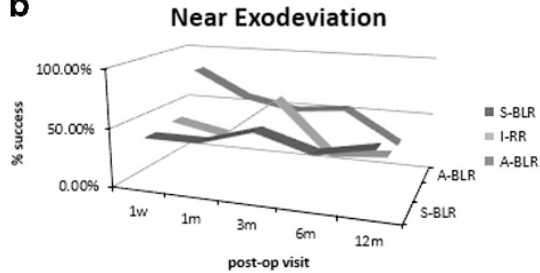

C

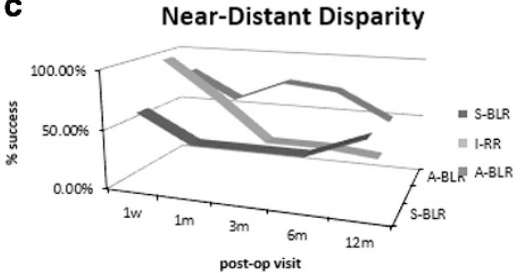

Figure 1 Graphical representation of the cumulative probabilities of success in the three groups. (a) Distant exodeviation, (b) near exodeviation, (c) near-distant disparity.

a

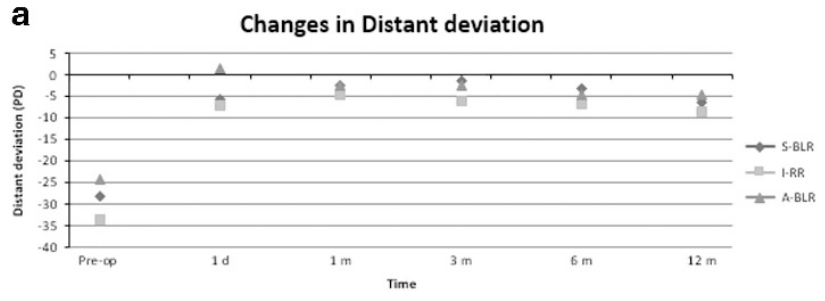

b

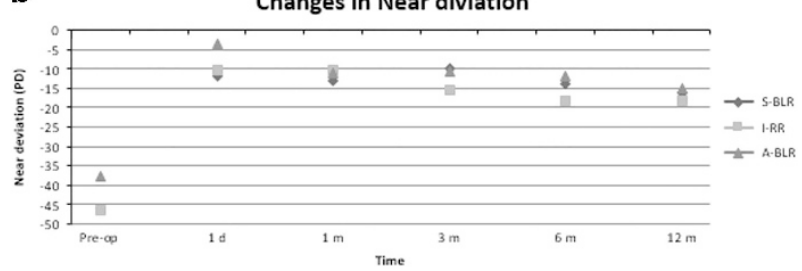

C

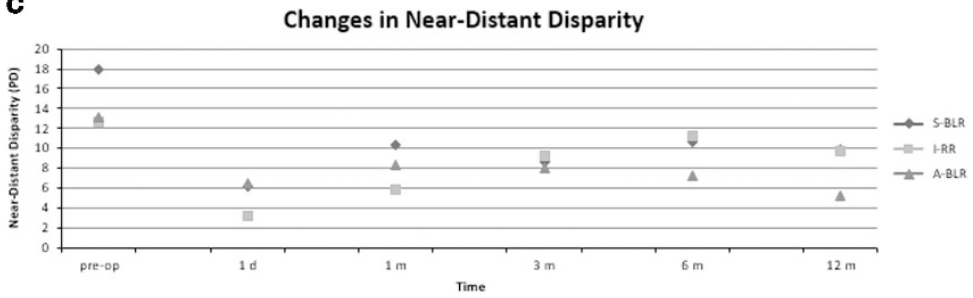

Figure 2 Graphical representation of the change in the angle of distant deviation (a), near deviation (b), and in near-distant disparity (c).

\section{Near exodeviation and near-distant disparity}

After surgery, the mean near exodeviation was reduced significantly in the three groups $(P<0.001$, paired $t$-test). At the last visit, the cumulative probability of success of exodeviation at near fixation were $50 \%, 30.4 \%$, and $27.2 \%$ in the S-BLR, I-RR, and A-BLR groups, respectively, a difference that was statistically insignificant $(P=0.233$; Figure $2 b)$. Of note, there were no recorded cases of postoperative overcorrection at near fixation in all the three groups (Table 2). After operation, the mean near-distant disparity showed a significant reduction only in the S-BLR and A-BLR groups $(P=0.002,0.007$, respectively), whereas it was nonsignificant in the I-RR group $(P=0.148)$. At the last postoperative visit, cumulative probability of success in the collapse of NDD were $59.09 \%, 30.4 \%$, and 50\% in the S-BLR, I-RR, and A-BLR 
groups, respectively, a difference that was found statistically nonsignificant $(P=0.142$; Figure $2 \mathrm{c})$.

\section{Near stereoacuity and AC/A ratio}

The mean near stereoacuity changed from $60.9 \pm 13.3$, $82.7 \pm 17.7,67.5 \pm 27.5$ arc sec. preoperatively to $36.2 \pm 12.1,59.9 \pm 14.6$, and $46.6 \pm 11.5$ arc sec. postoperatively in S-BLR, I-RR, and A-BLR groups, respectively. The difference between number of cases of improved stereopsis, stationary stereopsis, and decreased stereopsis among the three groups was statistically insignificant $(P=0.302,0.693$, and 0.105 , respectively; Table 1). Out of the 67 patients enrolled in this study, 13 patients had a normal AC/A ratio (average $3.4 \pm 2.7$ ) and 54 patients had a low $\mathrm{AC} / \mathrm{A}$ ratio (average $1.7 \pm 0.5$ ) before surgery. There was no statistical difference between $\mathrm{AC} / \mathrm{A}$ ratio among the three groups both preand postoperatively $(P=0.189$, and 0.214 , respectively; Table 1). However, the I-RR is the only procedure that had a significant impact on $\mathrm{AC} / \mathrm{A}$ ratio $(P=0.0005$, paired $t$-test).

\section{Complications}

Postoperative, three cases $(13.6 \%)$ in the slanting group developed overcorrection in the downgaze 'V- pattern' that persisted till the last postoperative visit. The average postoperative deviations in downgaze, upgaze, and primary position were 9.5 \pm 7.7 PD ET, 8.4 \pm 4.7 PD XT, and $2.4 \pm 4.1 \mathrm{PD} X \mathrm{X}$, respectively. In the same group, another case $(4.5 \%)$ developed significant postoperative A pattern with 16.2 $\pm 5.2 \mathrm{PD} \mathrm{XT}, 4.2 \pm 2.8 \mathrm{PD} \mathrm{XT}$, and $4.4 \pm 4.1 \mathrm{PD} X \mathrm{XT}$ in downgaze, upgaze, and primary position, respectively. In all those patients, the $\mathrm{A}-\mathrm{V}$ pattern was asymptomatic, that is, no diplopia, and therefore, no further actions were needed.

Cases with early postoperative overcorrection at distance were given the proper care and management (Table 2). Those cases were managed by full cycloplegic hypermetropic correction and alternate full-time patching until diplopia resolved. After 1 month, cases of nonresolving consecutive esotropia ( $>6 \mathrm{PD}$ ) and diplopia, $8 / 22(36.3 \%)$ in S-BLR, $1 / 23(4.3 \%)$ in I-RR, and 4/22 (18.1\%) in A-BLR groups were managed by temporary Fresnel press-on prisms. At 6 months postoperatively, all cases of persistent consecutive esotropia, 4/22 (18.1\%) in S-BLR group, and 6/22 (27.2\%) in A-BLR group were less than 15 PD (mean 5.6 $\pm 2.2 \mathrm{PD}$ ) and the base-out prisms were weaned off. At the last follow-up visit, all cases of consecutive esotropia in the S-BLR and I-RR groups showed complete resolution of esotropia, whereas in the A-BLR group 6/22 cases (27.2\%) showed persistent consecutive esotropia at distance (mean $4.3 \pm 1.4$ PD) and none of them required reoperation. Of note, there was no single case of consecutive esotropia at near fixation recorded in either group throughout the whole study period (Table 2).

\section{Discussion}

Surgical management of convergence insufficiency-type intermittent exotropia is still highly controversial. ${ }^{2}$ The variable success rate of MR resection, either uni- or bilateral, which ranged from 27 to $67 \%$, was highly motivating for patenting new surgical strategies. ${ }^{4-6,15-17}$ In our trial, we aimed to prospectively compare three different surgical techniques, namely, slanting LR recession, improved unilateral recession-resection and augmented LR recession. After 1 year follow-up, there was no statistical difference amid groups in correction of distant exodeviation, near exodeviation, and in collapse of near-distant disparity.

The concept of slanting recession and resection of horizontal recti was originally introduced in 1971 by Boyd et al for correction of significant A-V pattern strabismus. ${ }^{18}$ In 1997, Biedner ${ }^{19}$ used single slanted MR resection to treat three patients with CI-X(T) and recommended this procedure for patient with 10-20 PD XT at near with $\leq 10 \mathrm{PD}$ at distant. Snir et $a l^{8}$ in 1999 prospectively compared slanting LR recession (12 patients) with standard LR recession (6 patients) in treatment of CI-X(T), and they reported a success rate of $91.6 \%$ in the slanting group. The mean NDD was collapsed from 14 to 2.9 PD with no reported cases of early postoperative overcorrection or torsional effect. In 2012, In Song and Lee $^{20}$ retrospectively compared slanting BLR (17 patients) with classic BLR (14 patients) in CI-X(T) and reported a success rate of $35 \%$ in the slanting group compared with $7 \%$ in the control group with collapse of NDD from 10.2 to 4.7PD. In the current trial, the success rate of the slanting LR recession at the final visit was $77.2 \%$ at distance, $50 \%$ at near, and $59.09 \%$ for NDD that was collapsed from 17.9 to 9.8 PD. At the final visit, no patient showed overcorrection at distant or near fixation. However, three patients developed V pattern, while one patient developed A pattern strabismus. The surgical principle of the slanting technique could explain the occurrence of $\mathrm{V}$ pattern as weakening of lower part of the LR muscle more than its upper part could lead to overcorrection in the downgaze compared with upgaze. However, the occurrence of A pattern could not be explained by the same principle, which could be resulted from vertical instability or torsional effects induced by the slanting procedure. In the previous studies, postoperative $\mathrm{A}$ and $\mathrm{V}$ patterns were not recorded after slanting LR recession for $\mathrm{CI}-\mathrm{X}(\mathrm{T}), 8,20$ and this could be attributed to the large number of cases recruited in the current series. 
Kraft et al in 1995 introduced the improved formula for unilateral recession-resection in treatment of CI-X(T) in which the LR recession and the MR resection were biased to the distant and near deviations, respectively, and employed this formula on $14 \mathrm{CI}-\mathrm{X}(\mathrm{T})$ patients. ${ }^{12}$ All patients showed a decrease in their near deviation to $\leq 8 \mathrm{PD}$ and NDD to $\leq 6 \mathrm{PD}$. The NDD was collapsed from 11.9 to $1.7 \mathrm{PD}$, with $18 \%$ of patients developing distant overcorrection at the final visit. This surgical strategy then gained popularity and was studied in different successive clinical trials. ${ }^{2,11,21}$ Choi et al ${ }^{21}$ prospectively examined the improved unilateral recession-resection on 14 child with CI-X(T) and yielded $42.9 \%$ success rate after 1 year. The NDD was collapsed from 11.3 to 4.6 PD, with only one patient $(7 \%)$ showing persistent consecutive esotropia at the final visit. Yang and Hwang ${ }^{11}$ retrospectively compared improved recession-resection with augmented LR recession in different subtypes of CI-X(T) according to their response to diagnostic monocular occlusion and they concluded that the improved recession-resection procedure was significantly more successful than the augmented LR recession in CI-XT maintained after monocular occlusion (true and masked CI-XT subtypes). Wang et $a l^{2}$ prospectively compared improved unilateral recession-resection with unilateral and bilateral MR resection, and concluded that the improved recessionresection was better than the other two techniques in controlling distant and near deviation at 6 months postoperative but with high rate of early postoperative overcorrection. In the current trial, the success rate of I-RR after 1 year was $43.4 \%, 50 \%$, and $59.09 \%$ at distant, near, and in collapse of NDD, respectively, with very low rate of postoperative overcorrection (one case/23 (4\%) at 1 month that resolved conservatively in the subsequent visits). Interestingly, the unilateral improved recessionresection procedure in our trial is more associated with undercorrections contrary to overcorrections reported in previous trials. This discrepancy could be attributed to large preoperative angle of exodeviation at distant (33.7 PD) and at near (46.4 PD) in our trial compared with those in the previous ones (18.3 and 30.1 PD in Kraft's, 25.5 and 33.8 PD in Choi's, 22.6 and 34.6 PD in Wang's trials, respectively). Selecting patients with uniocular dominance and, therefore, with lower binocular potentials for the I-RR in our trial could be another contributing factor.

Bilateral LR recession augmented to near measurements was used in the study by Yang and Hwang ${ }^{11}$ and yielded a success rate of $61 \%$ in true CI-X(T) and $58 \%$ in masked CI-X(T). The incidence of immediate postoperative overcorrection requiring base-out prisms was recorded only in masked CI-X(T) $(8.3 \%)$. In the augmented group of our trial, the success rates at distant, near, and in NDD were $50 \%, 27.2 \%$, and $50 \%$, respectively. The incidence of postoperative overcorrection at distant was $36.3 \%(8 / 22)$ at first postoperative day and $27.2 \%(6 / 22)$ after 1 year. The difference in rates of postoperative overcorrection at the final visit between A-BLR and each of S-BLR and I-RR groups was statistically significant $(P=0.009$ and 0.007 , respectively, 'N-1' $\chi^{2}$-test). Patients with constant deviation and, therefore, lower fusional potentials are more in Yang and Hwang trial (44.6\%) compared with our trial $(22.7 \%)$, and this could be responsible for the high rate of immediate postoperative overcorrection in the augmented group in our trial (36.3\%) compared with Yang and Hwang trial (5.4\%).

This study reveals valuable information that can be applied in the management of CI-X(T). Compared with the previously published reports, the current trial recruited a large patient population (67 patients) and has a relatively long time follow-up (1 year). In addition to its prospective nature, the current trial also put three different surgical strategies in comparison. Another worthy point is that in many previous reports, the success was aimed at correction of distant deviation only, while in the current study, success was aimed at correction of distant, near deviations, and near-distant difference. Compared with previous trials concerning CI-X(T), and up to our knowledge, this is the only trial that studies the effect of each procedure on $\mathrm{AC} / \mathrm{A}$ ratio and, therefore, $\mathrm{AC} / \mathrm{A}$ ratio was measured in all patients in the three groups before operation and at the last follow-up visit. We have found that improved recession-resection was the only procedure that had a significant positive impact on $\mathrm{AC} / \mathrm{A}$ ratio, although the difference between the mean $\mathrm{AC} / \mathrm{A}$ ratio between the three groups was nonsignificant both before and after operation.

Overall, the comparison between the three different surgical techniques reveals important information that could benefit the management of CI-X(T). First, regarding the success rate in correction of distant exodeviation, the slanting recession technique achieved the highest values compared with the other two techniques, and this value, although nonsignificant, was very close to achieve statistical significance $(P=0.054)$. Similarly, the slanting recession was the technique that achieved the highest success rates in correction of near exodeviation and NDD, although these figures were statistically nonsignificant. However, such achievement of the slanting recession technique should be carefully weighed against its association with postoperative A and V patterns. Second, the difference in the success rate in correction of distant, near deviations, and correction of NDD between augmented recession and recession-resection was statistically insignificant. However, augmented recession was significantly associated with postoperative consecutive esotropia and diplopia. Finally, the improved 
recession-resection gave a relatively moderate success rate in correction of distant, near exodeviations, and neardistant difference. Giving the fact that postoperative undercorrection frequently associated with improved recession-resection is the simplest postoperative complication to deal with compared with overcorrection and $\mathrm{A}-\mathrm{V}$ patterns, the current study with its prospective nature, large number of patients, and relatively long time follow-up recommends the improved unilateral recession-resection technique for treatment of CI-X(T).

\section{Summary}

What was known before

- Convergence insufficiency intermittent $X(T)$ is a rare form of intermittent exodeviation.

- Surgical options for treatment of CI-X(T) are highly controversial.

- Unilateral rescession-resection biased to near and distant deviations is recently recommended by some authors.

\section{What this study adds}

- Slanting LR recessin achieved the highest success rate in correction of distant, near deviations, and NDD.

- However, it was associated with significant number of postoperative $\mathrm{V}$ and $\mathrm{A}$ pattern.

- Augmented LR recession is associated with significant number of postoperative consecutive esotropia and diplopia.

- Unilateral recession-resection gave a relatively moderate success rate and is associated with postoperative undercorrection.

\section{Conflict of interest}

The authors declare no conflict of interest.

\section{References}

1 Burian HM. Exodeviations: their classification, diagnosis and treatment. Am J Ophthalmol 1966; 62: 1161-1166.

2 Wang B, Wang L, Wang Q, Ren M. Comparison of different surgery procedures for convergence insufficiency-type intermittent in children. Br J Ophthalmol 2014; 98: 1409-1413.

3 Raab EL, Parks MM. Recession of the lateral recti; effect of preoperative fusion and distance-near relationship. Arch Ophthalmol 1975; 93: 584-586.
4 von Noorden GK. Resection of both medial rectus muscles in organic convergence insufficiency. Am J Ophthalmol 1976; 81: 223-226.

5 Nemet $\mathrm{P}$, Stolovich C. Biased resection of the medial recti: a new surgical approach to convergence insufficiency. Binocul Vis Strabismus Q 1990; 5: 213-216.

6 De Decker W, Baenge JJ. Unilateral medial rectus resection in the treatment of small-angle exodeviation. Graefes Arch Clin Exp Ophthalmol 1988; 226: 161-164.

7 Burian HM, Spivey BE. The surgical management of exodeviations. Am J Ophthalmol 1956; 59: 603-620.

8 Snir M, Axer-Siegel R, Shalev B, Sherf I, Yassur Y. Slanted lateral rectus recession for exotropia with convergence weakness. Ophthalmology 1999; 106: 992-996.

9 Choi MY, Hwang JM. The long-term result of slanted medial rectus resection in exotropia of the convergence insufficiency type. Eye 2006; 20: 1279-1283.

10 Choi DG, Rosenbaum AL. Medial rectus resection(s) with adjustable suture for intermittent exotropia of the convergence insufficiency type. J AAPOS 2001; 5: 13-17.

11 Yang HK, Hwang JM. Surgical outcomes in convergence insufficiency-type exotropia. Ophthalmology 2011; 118: 1512-1517.

12 Kraft SP, Levin AV, Enzenauer RW. Unilateral surgery for exotropia with convergence weakness. J Pediatr Ophthalmol Strabismus 1995; 32: 183-187.

13 Jackson JH, Arnoldi K. The gradient AC/A ratio: what's really normal? Am Orthopt J 2004; 54: 125-132.

14 Adams WE, Leske DA, Hatt SR, Holmes JM. Defining real change in measures of stereoacuity. Ophthalmology 2009; 116: 281-285.

15 Haldi BA. Surgical management of convergence insufficiency. Am Orthopt J 1978; 28: 106-109.

16 Hermann JS. Surgical therapy for convergence insufficiency. J Pediatr Ophthalmol Strabismus 1981; 18: 28-31.

17 Kushner B. Exotropic deviations: a functional classification and approach to treatment. Am Orthopt J 1988; 38: 81-93.

18 Boyd TAS, Leitch GT, Budd GE. A new treatment for 'A' and ' $\mathrm{V}$ ' patterns in strabismus by slanting muscle insertions. A preliminary report. Can J Ophthalmol 1971; 6: 170-177.

19 Biedner B. Treatment of convergence insufficiency by single medial rectus muscle slanting resection. Ophthalmic Surg Lasers 1997; 28: 347-348.

20 Song IJ, Lee SG. The effect of bilateral slanted lateral rectus recession in exotropia with near-far disparity. J Korean Ophthalmol Soc 2012; 53: 311-315.

21 Choi MY, Hyung SM, Hwang JM. Unilateral recessionresection in children with exotropia of the convergence insufficiency type. Eye 2007; 21: 344-347. 